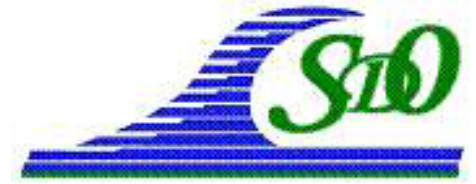

\title{
Influence de la gestion hydraulique sur la dégradation des milieux du Lac de Grand-Lieu : éléments de discussion
}

\author{
Eric MERCIER ${ }^{1}$, Martin SANCHEZ ${ }^{1}$
}

1. Université de Nantes, Faculté des Sciences,

Laboratoire de Planétologie et Géodynamique (CNRS UMR-6112),

2, rue de la Houssinière, BP 92208, 44322 Nantes cédex 3, France.

eric.mercier@univ-nantes.fr; martin.sanchez@univ-nantes.fr

\section{Résumé :}

Le Lac de Grand-Lieu, situé au cœur d'une vaste zone humide de l'ouest de la France, voit sa biodiversité menacée par, notamment, un processus d'érosion. Nous montrons que la gestion hydraulique qui est pratiquée surtout depuis 1985 a une part de responsabilité dans cet état de fait. Deux paramètres de cette gestion sont examinés : les niveaux estivaux du Lac et les pointes de débit qui résultent du respect du règlement d'eau.

\section{Mots-clés :}

Lac de Grand-Lieu - Erosion lacustre - Gestion hydraulique - Crise érosive - Crue

\section{Introduction}

Le Lac de Grand-Lieu constitue le cœur d'une vaste zone humide située à quelques kilomètres au Sud-Ouest de Nantes (44). Ce Lac, et la zone humide qui l'entoure, constituent des milieux écologiques remarquables, et en toute logique, ils sont protégés par un arsenal de dispositifs juridiques (ZNIEFF, Site inscrit, Site classé, Réserve de chasse, ZPS, Natura 2000, Ramsar, Réserve Naturelle; STRUILLOU et al., 2006). Néanmoins on assiste, depuis quelques décennies, à la dégradation de nombreux milieux écologiques dont certains parmi les plus remarquables. Cette dégradation se traduit notamment par une érosion des roselières au profit du système lacustre ( $15 \mathrm{ha} / \mathrm{an}$ en moyenne depuis 2000 : MERCIER \& SANCHEZ, sous presse) et, au sein de ce dernier, par l'augmentation des surfaces d'eau libre, par la multiplication des blooms alguaires et par la transformation des herbiers palustres (figure 1).

Les causes de cette évolution sont complexes et objets de nombreux débats, ainsi la probable altération de la qualité des eaux provenant du bassin versant, et le rôle des espèces invasives (ragondins Myocastor coypus, écrevisses de Louisiane Procambarus clarkii,) sont souvent mises en avant. D'autres paramètres pourraient également jouer, comme la modification du peuplement ichtyologique, suite à l'évolution des pratiques de la pêche professionnelle, ou l'abandon de l'exploitation (fauchage) des marais. Nous voudrions montrer ici que la gestion hydraulique du Lac est également un paramètre à prendre en compte, à la fois comme co-responsable de la dégradation du milieu, mais 
Thème 2 - Dynamique sédimentaire et transports des particules

surtout comme levier potentiel d'action dans une démarche de reconquête de la qualité de cet espace naturel.
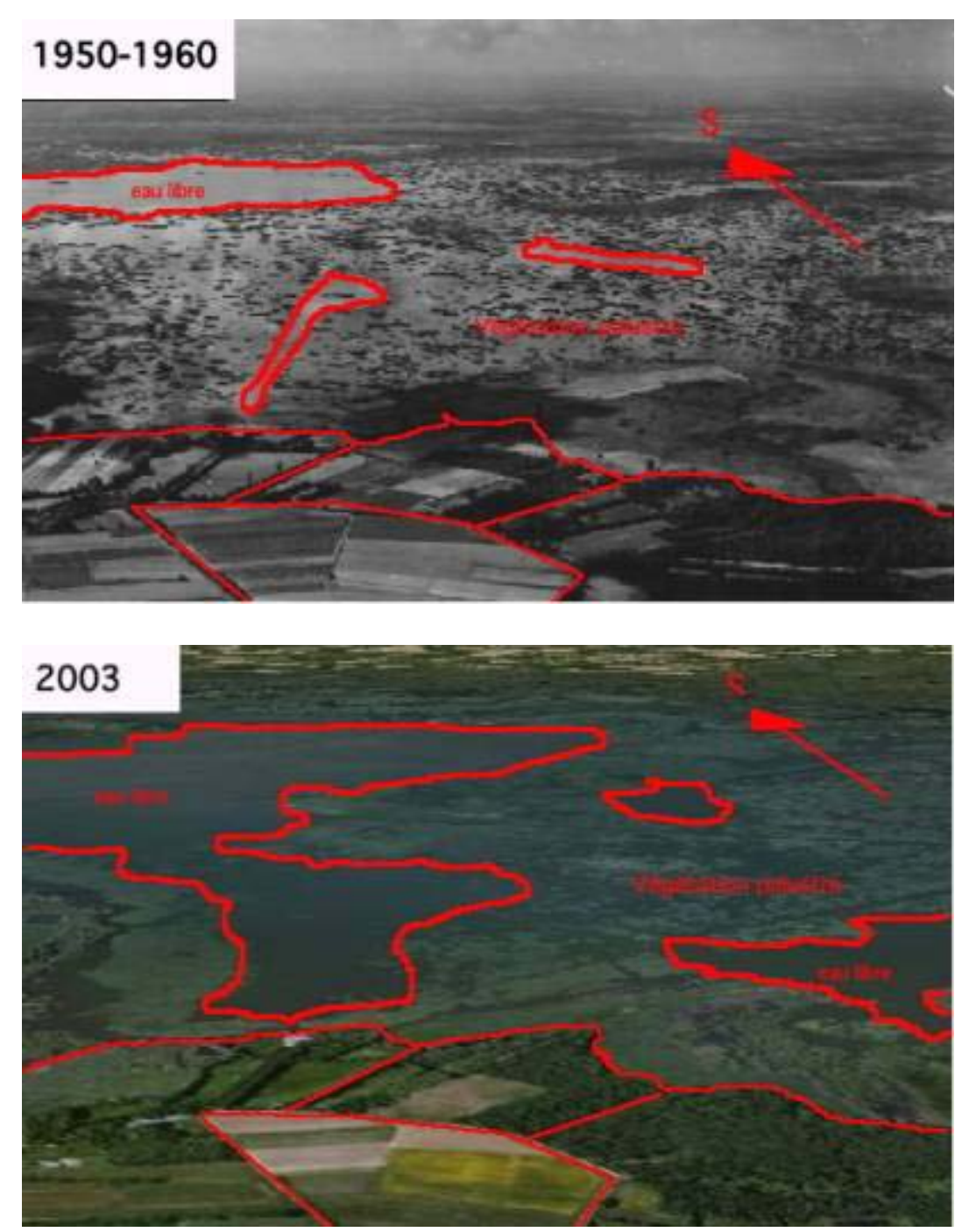

Figure 1. Comparaison d'une photographie aérienne oblique ancienne et d'une scène GoogleEarth (2003) qui montre l'augmentation de l'eau libre et le remplacement des herbiers à Scirpes lacustres (Scirpus lacustri)s, Limnanthèmes jaunes (Nymphoides peltata), et châtaignes d'eau (Trapa natans) par des herbiers à nénuphars blancs (Nymphaea candida) et nénuphars jaunes (Nuphar luteum).

\section{Contexte hydraulique}

La zone humide du Lac de Grand-Lieu (bassin versant de $840 \mathrm{~km}^{2}$ ) est alimentée par deux rivières (la Boulogne et l'Ognon) qui ont un régime très irrégulier. Cette variation des apports se traduit par une grande fluctuation du niveau du Lac (figure 2A) et de la surface d'inondation (2000 à 5000 hectares selon la saison). La vidange du Lac vers 
l'estuaire de la Loire par l'intermédiaire de la rivière Acheneau est contrôlée par l'écluse de Bouaye en respectant un règlement d'eau (cotes actuelles sur la figure 2A).

En fait, cette situation est relativement récente et on peut décrire l'évolution de la gestion depuis un siècle de la façon, suivante (figure 3 et GUILLOU et al., 2008) :

- avant 1958, le niveau du lac au printemps était très variable et reflétait l'ampleur des crues hivernales et la capacité (faible) que possédait l'Acheneau à évacuer les débits ;

- sur la période 1958-1964, un profond recalibrage de l'Acheneau a été réalisé si bien que les cotes estivales du lac se sont écroulées. Le propriétaire du lac a obtenu alors la construction de l'écluse de Bouaye qui commande le niveau du Lac ;

- à partir de 1964, cette écluse a permis de remonter les niveaux printaniers moyens en supprimant les étiages estivaux. Par ailleurs, c'est à cette époque qu'a été construit le canal Guerlain qui constitue un raccourci hydraulique au niveau de l'exutoire du Lac ;

- à partir de 1985, une gestion plus rigoureuse de l'écluse permet aussi d'éliminer très tôt les volumes résiduels des crues d'hiver ;

- sur la période 1995-actuelle, les niveaux printaniers ont de nouveau été remontés $\left(+0,50 \mathrm{~m}\right.$ au $1^{\mathrm{er}}$ mai par exemple). Suite à des conflits importants avec les riverains, des modérations de la hausse des niveaux ont été appliquées successivement en 1996 et 2004.

A

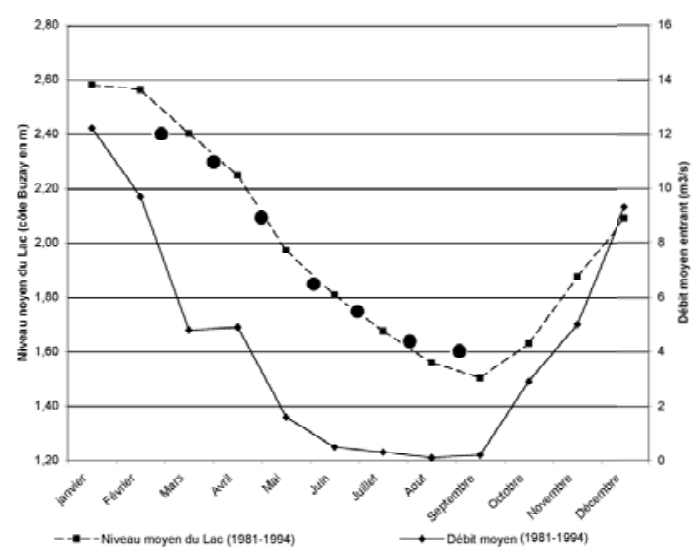

B

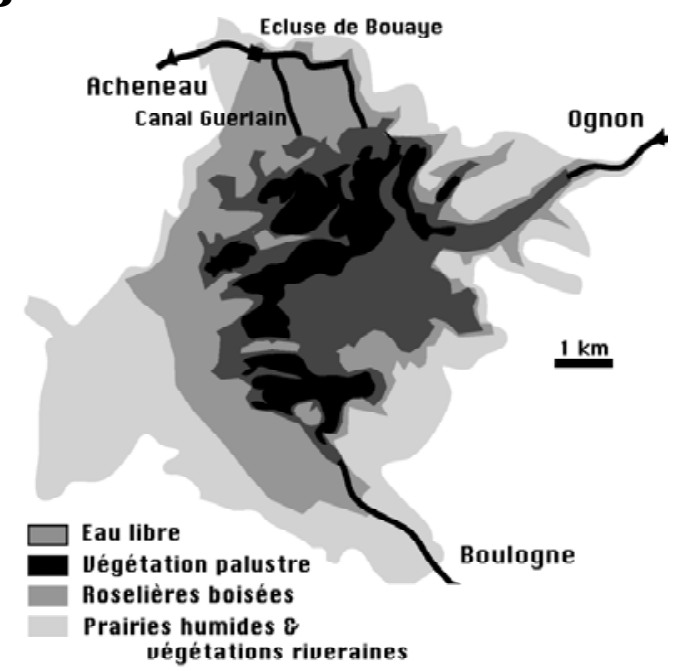

Figure 2. L'hydraulique du Lac de Grand-Lieu (d'après MERCIER \& SANCHEZ, sous presse). A : Moyenne interannuelle (1981-1994) du débit entrant et niveau moyen du lac dans la période 1981-1994 (en cote "Buzay" $=N G F(I G N$ 1969) - 0,45 m). Les points noirs indiquent le règlement d'eau actuel. B : Carte des milieux et des ouvrages hydrauliques. 


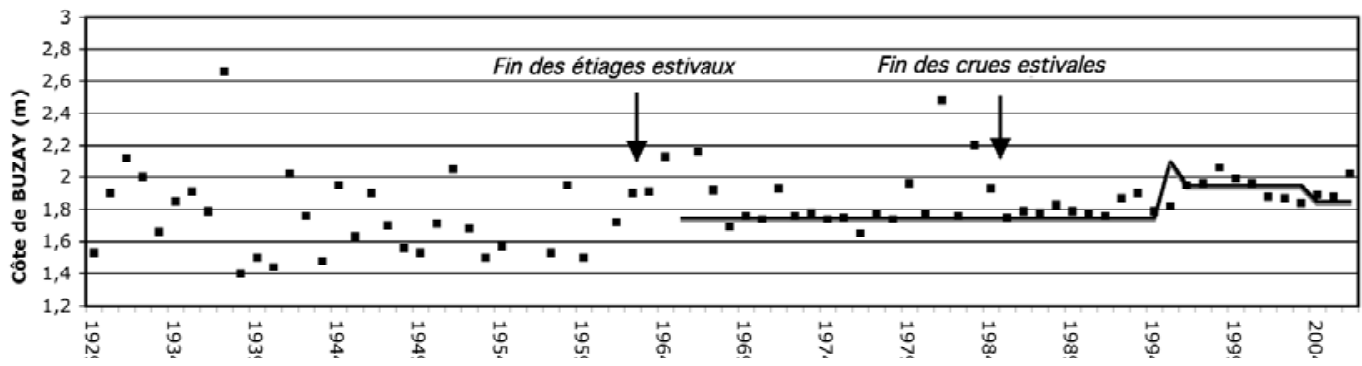

Figure 3. Cote du lac au $1^{\text {er }}$ juin depuis 1929. Le trait gras continu indique l'évolution du règlement d'eau appliqué. On remarque deux ruptures dans la gestion qui correspondent à la construction de l'écluse de Bouaye, au début des années 1960, puis à une plus grande rigueur dans le respect du règlement d'eau (1985).

\section{Influence de la gestion de niveaux}

REEBER (2000), DUPONT (2003) et MERCIER \& SANCHEZ (sous presse) ont déjà montré que la gestion actuelle des niveaux (niveaux printanier et estival haut, et absence de variabilité interannuelle à une date donnée) avait un impact négatif sur l'écosystème. Cet impact se manifeste notamment par une baisse de la densité, de la diversité et de la productivité des macrophytes flottants (PAILLISSON \& MARION, 2006), (figure 1). Par ailleurs, les microphytes (plancton) qui sont en concurrence avec les macrophytes dans l'écosystème, tirent un bénéfice de cette évolution ce qui contribue à favoriser les blooms alguaires. Enfin, la diminution des populations de macrophytes a une influence directe sur la vitesse des courants, sur la génération et l'amplification des vagues et sur la remise en suspension des sédiments et donc, sur l'érosion. Mais le principal impact de la gestion hydraulique sur l'érosion est selon nous, en relation avec la gestion des débits.

\section{L'érosion du Lac de Grand-Lieu}

L'analyse des photographies aériennes montre que la zone lacustre s'accroît depuis le milieu des années 1970 alors qu'elle régressait antérieurement (45 ha/an). Cette rupture dans l'évolution naturelle s'accélère, l'érosion est actuellement de 15 ha/an en moyenne alors qu'elle ne dépassait pas 5 ha/an à la fin des années 1970 (MERCIER \& SANCHEZ, sous presse). Il est significatif de noter que c'est tout d'abord les rivages situés à proximité du canal Guerlain qui ont été affectés par l'érosion (vers 1976), le phénomène n'a intéressé l'ensemble des rives du Lac que quelques années après. Parallèlement le Canal Guerlain, qui en 1964 avait été creusé à 3 m de large, s'est spontanément élargi pour faire maintenant $25 \mathrm{~m}$ au miroir, et représenter $80 \%$ des capacités de vidange du Lac. Cette érosion des bords est couplée à une érosion du fond bien mise en évidence par des campagnes de bathymétrie réalisées par le gestionnaire du site: la Société Nationale de Protection de la Nature (SNPN). Ces campagnes 
concernent plus de 2000 points mesurés deux fois à 7 ans d'intervalle; elles ont permis d'évaluer la vitesse d'approfondissement qui est de l'ordre de $0,5 \mathrm{~mm} / \mathrm{an}$. Nous avons montré (MERCIER \& SANCHEZ, sous presse) que cette érosion du fond et des bords du Lac était due à un déséquilibre récent entre les apports de MES (5000 à $10000 \mathrm{t}$, CEMAGREF in JIGOREL, 1992), et l'exportation (1,5 à 2 fois plus importante). Deux processus sont en cause : (1) la remise en suspension des sédiments par l'effet du vent et (2) l'existence de forts courants de vidange. Les conditions naturelles du Lac (grande taille, proche de l'océan, peu profond, sédiments fins et peu cohésifs, ...) favorisent fortement la remise en suspension (supérieur à $200 \mathrm{mg} / \mathrm{l}$ de MES en sortie en cas de coup de vent, BORET, 2005). Nous nous proposons de montrer que la gestion actuelle du Lac quant à elle, favorise les forts courants de vidange et donc l'érosion.

\section{Gestion hydraulique et courants de vidange}

A partir du milieu des années 1980, le meilleur respect des cotes fixées par le règlement d'eau a nécessité des vidanges du Lac de plus en plus rapides, la figure 4 montre que, si avant 1985 des baisses de $8 \mathrm{~cm}$ en une journée étaient exceptionnelles, après cette date, cela devient plus fréquent.

Cette augmentation de la baisse journalière du niveau se traduit par une augmentation du débit de vidange. Le débit de sortie à l'écluse de Bouaye en période de vidange du Lac (baisse de niveau) est donné par :

$Q s=Q e+\Delta V l / d t$

avec $Q s$ : débit de sortie $\left(\mathrm{m}^{3} / \mathrm{s}\right), Q e$ : débit d'entrée $\left(\mathrm{m}^{3} / \mathrm{s}\right)$ et $\Delta V l:$ la variation du volume stocké dans le Lac.

La variation du volume stocké dans le Lac pour une chute de niveau donnée peut être calculée grâce à l'hypsométrie de la zone humide en y incluant le cours inférieur de la Boulogne et celui de l'Ognon (figure 1).

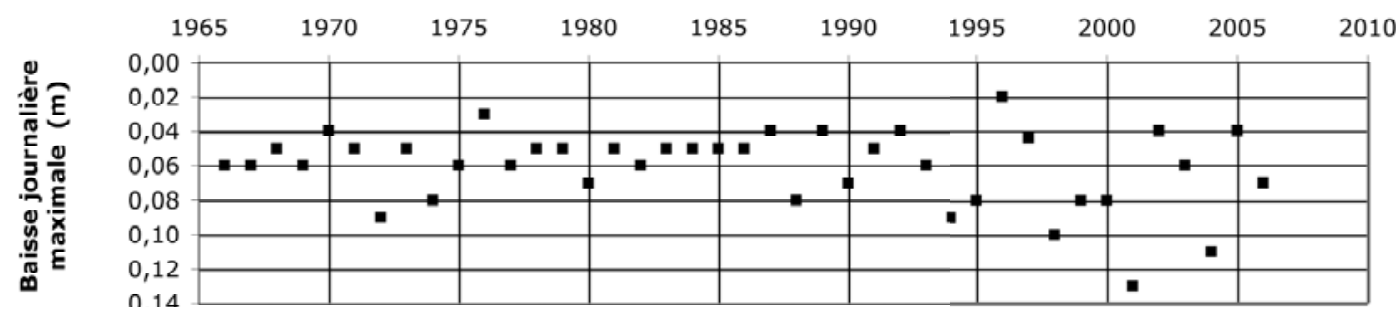

Figure 4. Baisse journalière maximale du niveau du Lac sur chaque année depuis 1966.

On constate une augmentation des records annuels depuis le milieu des années 1980. 


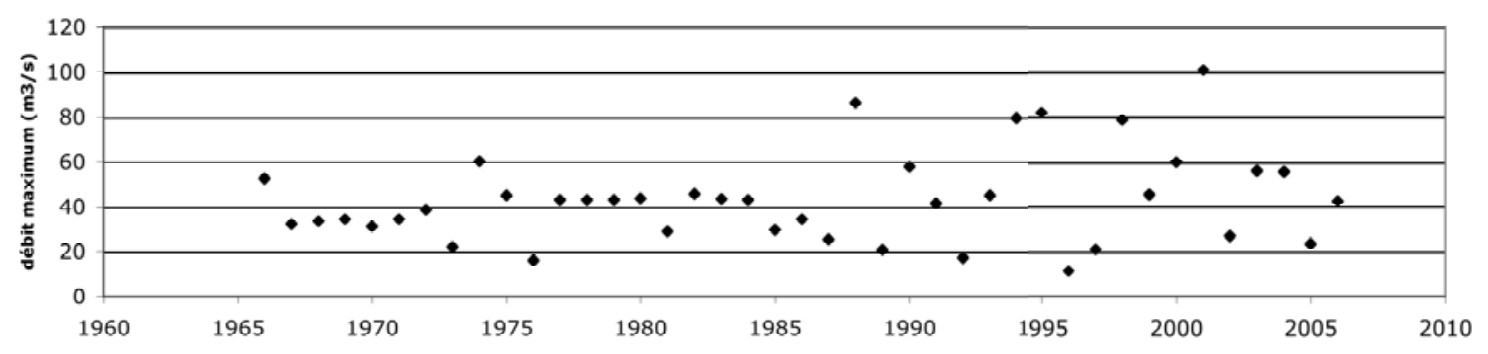

Figure 5. Maxima annuels des débits journaliers en sortie de Lac (terme $\Delta V l / d t$ en $\mathrm{m}^{3} / \mathrm{s}$ ).

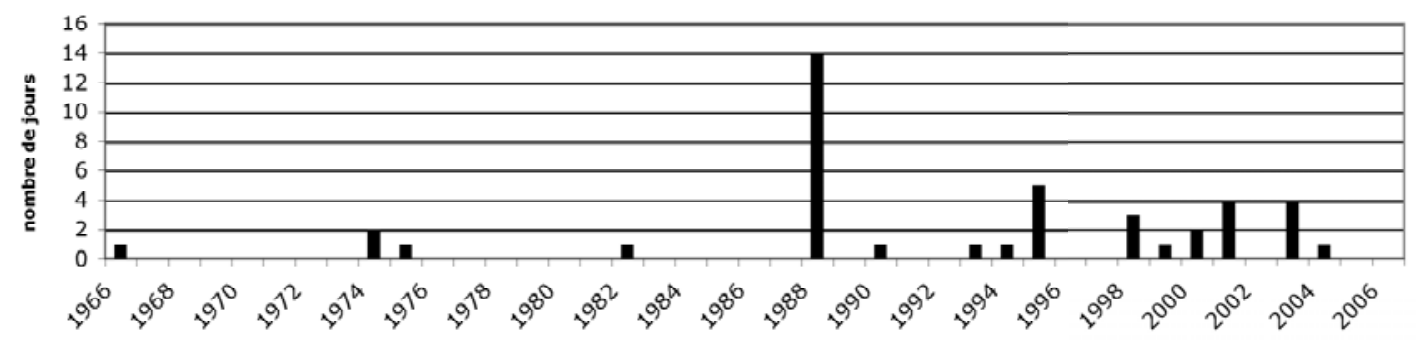

Figure 6. Nombre de jours par an où le débit journalier est supérieur à $45 \mathrm{~m}^{3} / \mathrm{s}$.

L'analyse des chroniques disponibles montre que les pointes de vidange, qui interviennent suite à une manœuvre de vannes à l'initiative du gestionnaire, ont lieu en décalage par rapport aux crues des contributaires. En période de pointe de vidange, le terme $Q e$ peut donc être considéré comme négligeable devant l'autre. Les figures 5 et 6 , ont été établies en tenant compte de cette approximation. Ces figures permettent de constater que, depuis 1985, non seulement les débits de pointe sont significativement plus importants, mais aussi qu'ils sont plus fréquents.

Il est donc établi que l'introduction de la gestion actuelle a augmenté la fréquence et l'importance des courants de vidange. Nous voudrions souligner que ces courants peuvent avoir une influence significative sur le bilan sédimentaire. Pour cette démonstration, nous allons nous intéresser, à titre d'exemple, à la période des 27, 28 et 29 janvier 1998. Pendant cette période, le hasard a voulu qu'il y ait conjonction d'une forte vidange du Lac (perte de 0,21 m en 72 heures, avec un débit de sortie qui a atteint une moyenne journalière de $78 \mathrm{~m}^{3} / \mathrm{s}$ le 29 janvier) et de vents forts d'E-NE atteignant $30 \mathrm{~km} / \mathrm{h}$. Pendant ces trois jours, le Lac a perdu 19,5\% de l'eau stockée $\left(15,310^{6} \mathrm{~m}^{3}\right)$ et la vitesse dans le canal Guerlain a atteint une moyenne de $1 \mathrm{~m} / \mathrm{s}$ sur la journée du 29 janvier. Si l'on extrapole les données de BORET (2005) et que l'on retient une MES en sortie du Lac de $200 \mathrm{mg} / \mathrm{l}$ pendant cette période de 3 jours : on obtient une exportation de plus de $3000 \mathrm{t}$ de MES (1400 t la journée du 29 janvier), soit de 30 à $60 \%$ des apports annuels de MES. 


\section{Discussion et conclusion}

Le calcul précédent souffre d'un certain nombre d'approximations, notamment sur la valeur de la MES qui est incertaine. Néanmoins, il illustre l'influence, sur le bilan sédimentaire, des périodes de vidange rapide, qui ont été introduite à partir de 1985 pour respecter, à certaines dates, des niveaux fixés par le règlement d'eau (figure 6). Il n'est pas possible actuellement d'évaluer la part qu'ont ces périodes exceptionnelles dans le bilan sédimentaire, et notamment il n'est pas possible d'établir si leur suppression suffirait à équilibrer le bilan. En fait cela est peu probable car la tendance érosive actuelle avait commencé dans le canal Guerlain (probablement dès sa création) et dans le Lac (en 1975) avant la mise en place de cette gestion (1985). Une amélioration est néanmoins souhaitable. Cette amélioration pourrait simplement consister à interdire la vidange du lac en période de forte turbidité et/ou par fort vent, ce qui pourrait conduire à différer de quelques jours (semaines?) l'application du règlement d'eau. En fait, la maîtrise de l'érosion (l'objectif étant de diminuer de $33 \%$ à $50 \%$ l'érosion lacustre), passe probablement par une redéfinition plus ambitieuse de la gestion hydraulique du Lac et, éventuellement, par des aménagements de l'exutoire. Vu les conflits antérieurs qui se sont focalisés sur ces questions, et la lenteur des évolutions naturelles, une démarche d'amélioration de la gestion par tâtonnement ne paraît ni souhaitable, ni même réaliste. Notre laboratoire universitaire a proposé de réaliser, dans le cadre d'un appel d'offre de la Région Pays de la Loire, un modèle numérique de l'évolution hydro-sédimentaire du Lac (projet "granlieu »; P. Vacher, E. Mercier, M. Sanchez et V. Turpin). La réalisation de ce modèle passe par l'acquisition et le suivi de nombreux paramètres physiques qui nécessiteront l'instrumentation du Lac en complément des stations de mesures gérées par le SAGE de Grand-Lieu et par la "Compagnie d'exploitation des ports". Nous projetons de tester, avec ce modèle numérique, divers scenarii basés sur la gestion des débits et non plus sur celle des niveaux. Ce type de gestion irait dans le sens d'une limitation de l'érosion, mais également dans le sens d'une variabilité interannuelle. Ainsi, un haut niveau hivernal se traduirait probablement par une vidange plus longue, et donc des niveaux printaniers plus hauts; et un hiver sec, par un étiage précoce et des niveaux estivaux plus bas. Une telle variabilité interannuelle, qui réintroduirait un peu de "naturalité" dans l'hydraulique du Lac, est souhaitée à la fois par REEBER (2000) pour l'avifaune et DUPONT (2003) pour la végétation.

\section{Références}

BORET P. (2005). Bathymétrie de la zone centrale du lac de Grand-Lieu. SNPN, Paris. DUPONT P. (2003). L'évolution de la flore et de la végétation du lac de Grand-Lieu (Loire Atlantique) et de ses ceintures : situation actuelle, problèmes de conservation et de gestion. Bulletin de la Société Botanique du Centre-Ouest, 34, pp 3-64. 
Thème 2 -Dynamique sédimentaire et transports des particules

GUILLOU J.J., MERCIER E., SANCHEZ M. (2008). Influence des aménagements hydrauliques sur l'évolution des niveaux d'eau dans le Lac de Grand-Lieu depuis le XVIIIe siècle. Bull Soc. Historiens pays Retz, (27), pp 21-30.

JIGOREL A. (1992). Étude sédimentologique du lac de Grand-Lieu. Rapport INSA de Rennes.

MERCIER E., SANCHEZ M. (sous presse). Essai de bilan sédimentation/érosion du Lac de Grand-Lieu (Loire Atlantique) : conséquences pour la gestion du milieu naturel. Rev. Ecologie (Terre Vie).

PAILLISSON J.M., MARION L. (2006). Can small water level fluctuations affect the biomass of Nymphaea alba in large lakes? Aquatic Botany, 84, pp 259-266.

REEBER S. (2000). Impact du relèvement du niveau d'eau du lac de Grand-Lieu sur l'avifaune. SNPN, Paris.

STRUiLlou J.F., MENANTEAU L., CHADENAS C., POURINET L. (2006). Protection du patrimoine naturel et développement durable dans l'estuaire de la Loire. Le cas du lac de Grand-Lieu. Colloque International Interactions Nature-Société : analyses et modèles, La Baule (Loire-Atlantique - France) 3-6 mai 2006, 7 p. 